\author{
А. А. Аракелянц ${ }^{1}$, кандидат медицинских наук \\ T. Е. Морозова, доктор медицинских наук, профессор \\ Е. А. Барабанова, кандидат медицинских наук \\ Е. О. Самохина, кандидат медицинских наук \\ ФГАОУ ВО Первый МГМУ им. И. М. Сеченова Минздрава России, Москва, Россия
}

Резюме. У здоровой женщины при нормально протекающей беременности структурно-функциональные изменения сердца носят адаптивный характер, выражены незначительно и полностью нормализуются после родов. У беременной с теми или иными заболеваниями сердца адаптационные возможности снижены, поэтому на любом этапе беременности могут возникать патологические изменения, осложняющие ее течение и приводящие к неблагоприятным исходам. Кроме того, при любой беременности возможно развитие новых заболеваний сердца, которые могут быть фатальными. Вот почему эхокардиографическая оценка структурно-функциональных изменений сердца является необходимой как при нормальной беременности, так и на фоне сопутствующих сердечно-сосудистых заболеваний.

Ключевые слова: беременность, эхокардиография, пороки сердца, перипартальная кардиомиопатия, сердечно-сосудистый риск. Для цитирования: Аракелянц А. А., Морозова Т. Е., Барабанова Е. А., Самохина Е. О. Структурно-функциональные изменения сердца при беременности у женщин с сердечно-сосудистыми заболеваниями // Лечащий Врач. 2021; 7 (24): 18-23. DOI: 10.51793/OS.2021.24.7.004

\title{
Structural and functional heart features in pregnancy with concomitant cardiovascular diseases
}

A. A. Arakelyants 1, T. E. Morozova, E. A. Barabanova, E. O. Samokhina

I. M. Sechenov First Moscow State Medical University of the Ministry of Health of the Russian Federation, Moscow, Russia

Abstract. In a healthy woman with a normal pregnancy, structural and functional changes in the heart are adaptive in nature, are slight and are completely normalized after childbirth. A pregnant woman with heart diseases has reduced adaptive capacity, therefore, at any stage of pregnancy, pathological changes can occur that complicate its course and lead to adverse outcomes. In addition, any pregnancy may develop new heart diseases, which can be fatal. That is why an echocardiographic assessment of structural and functional changes in the heart is necessary both during normal pregnancy and in the background of concomitant cardiovascular diseases.

Keywords: pregnancy; echocardiography; heart diseases; peripartal cardiomyopathy; cardiovascular risk.

For citation: Arakelyants A. A., Morozova T. E., Barabanova E. A., Samokhina E. O. Structural and functional heart features in pregnancy with concomitant cardiovascular diseases // Lechaschy Vrach. 2021; 7 (24): 18-23. DOI: 10.51793/OS.2021.24.7.004

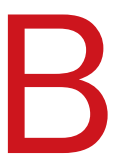

ажное значение эхокардиографии (ЭхоКГ) в обследовании беременных для выявления структурно-функциональных изменений сердца сегодня не вызывает сомнений, а ее место определено рядом современных клинических рекомендаций. Так, в 2018 г. были опубликованы пересмотренные рекомендации Европейского общества кардиологов (ESC) по ведению пациенток с сердечно-сосудистыми заболеваниями (СС3) во время беременности [1], в которых подтверждены, а также уточнены основные положения по применению ЭхоКГ в этой группе женщин. Чуть раньше были утверждены российские национальные рекомендации «Диагностика и лечение сердечно-сосудистых заболеваний при беременности» [2], обобщившие отечественные и наиболее значимые зарубежные клинические рекомендации за 2011-2017 гг.

\footnotetext{
${ }^{1}$ Контактная информация: nxrrimma@mail.ru
}

Кроме того, в последние годы вышли системные обзоры и метаанализы, посвященные внутрисердечной гемодинамике, ремоделированию сердца, сердечно-сосудистой патологии у беременных, в той или иной мере рассматривающие вопросы, связанные с анализом и интерпретацией данных, получаемых при ЭхоКГ. В связи с этим целью данной статьи является рассмотрение ключевых моментов, знание которых необходимо врачам так называемой «мультидисциплинарной команды» (кардиолог, акушер-гинеколог, анестезиолог-реаниматолог и другие привлекаемые специалисты) для оценки состояния сердца при планировании, во время и после беременности.

\section{Определение сердечно-сосудистого риска при планировании беременности}

На сегодня, по данным статистики здравоохранения и исходя из клинической практики, установлено, что в целом 
сердечно-сосудистая патология выявляется не менее чем у $10 \%$ беременных и стойко занимает второе, после анемии, место среди экстрагенитальной патологии [2, 3]. Это артериальная гипертония (АГ), врожденные и приобретенные пороки сердца (ВПС и ППС соответственно), нарушения ритма сердца, кардиомиопатии и некоторые другие заболевания. Характер болезни и функциональное состояние сердца определяют течение беременности, риск осложнений, а иногда и противопоказания для беременности. В связи с этим при планировании беременности целесообразна оценка сердечно-сосудистого материнского риска.

Определение риска у женщин детородного возраста с СC3 следует проводить по модифицированной классификации Всемирной организации здравоохранения (ВО3) (табл. 1) [1]. Наряду с этим риск следует уточнить по наличию предикторов осложнений, значение которых было доказано в исследова- ниях CARPREG, ZAHARA и ROPAC. Данные предикторы риска осложнений у матери с кардиоваскулярной патологией изложены в табл. 2.

Как видно из приведенных таблиц, и классификация ВОЗ, и перечень предикторов осложнений в значительной степени базируются на выявляемых и рассчитываемых при ЭхоКГ структурно-морфологических признаках поражения сердца и аорты, дисфункции желудочков, давления в легочной артерии. Так, например, при болезнях аорты (в эту группу включают бикуспидальный аортальный клапан, синдромы Марфана, Элерса-Данлоса и другие) при отсутствии ее дилатации материнский риск считают низким, но его оценивают как очень высокий при дилатации свыше 45-50 мм в зависимости от патологии. По совокупности признаков, указывающих на возможность диссекции аорты, беременность противопоказана. Такое же значение имеют количественные ЭхоКГ-показатели,

\begin{tabular}{|c|c|}
\hline \multicolumn{2}{|c|}{$\begin{array}{l}\text { Модифицированная классификация ВОЗ сердечно-сосудистого риска материнской смертности и увеличения } \\
\text { заболеваемости / Modified WHO classification of cardiovascular risk of maternal mortality and increased morbidity } 1\end{array}$} \\
\hline $\begin{array}{l}\text { Риск В03 I } \\
\text { (отсутствует) }\end{array}$ & $\begin{array}{l}\text { - Неосложненные, слабо или умеренно выраженные: } \\
\text { о стеноз легочной артерии; } \\
\text { о открытый артериальный проток; } \\
\text { о пролапс митрального клапана } \\
\text { - Успешно оперированный порок сердца: } \\
\text { о септальные Аефекты; } \\
\text { о открытый артериальный проток; } \\
\text { о аномальный Аренаж легочных вен } \\
\text { - Изолированные предсердные или желудочковые экстрасистолы }\end{array}$ \\
\hline $\begin{array}{l}\text { Риск В0З ІІ } \\
\text { (низкий) }\end{array}$ & $\begin{array}{l}\text { - Неоперированный септальный дефект } \\
\text { - Коррегированная тетрада Фалмо } \\
\text { - Большинство аритмий (наАжелудочковые аритмии) } \\
\text { - Синдром Шерешевского-Тёрнера без дилатации аорты }\end{array}$ \\
\hline $\begin{array}{l}\text { Риск В03 ІІ-ІІІ } \\
\text { (промежуточный) }\end{array}$ & $\begin{array}{l}\text { - Аегкая Аисфункция левого желудочка (^Ж) - фракция выброса ^Ж > 45\% } \\
\text { - Гипертрофическая кардиомиопатия } \\
\text { - Заболевания естественных или биологических клапанов, не рассматриваемые как риск I или IV (легкий митральный } \\
\text { - стеноз, умеренный аортальный стеноз) } \\
\text { - Аиндром Марфана и Аругие наслеАственные болезни грудной аорты без дилатации аорты } \\
\text { - Аиаметр аорты < } 45 \text { мм при Авустворчатом клапане аорты } \\
\text { - Оперированная коарктация аорты } \\
\text { - Аефект преАсердно-желудочковой перегородки }\end{array}$ \\
\hline $\begin{array}{l}\text { Риск В03 III } \\
\text { (значительный) }\end{array}$ & 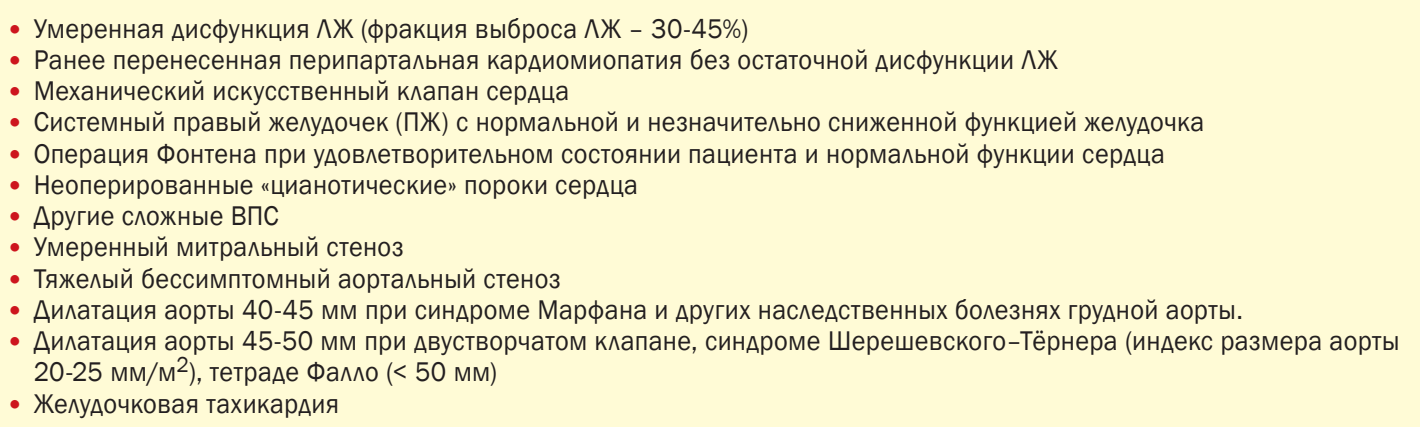 \\
\hline $\begin{array}{l}\text { Риск ВОз IV } \\
\text { (крайне высокий } \\
\text { риск; беременность } \\
\text { противопоказана; } \\
\text { в случае наступившей } \\
\text { беременности } \\
\text { необходимы врачебные } \\
\text { консультации } \\
\text { о ее прерывании) }\end{array}$ & 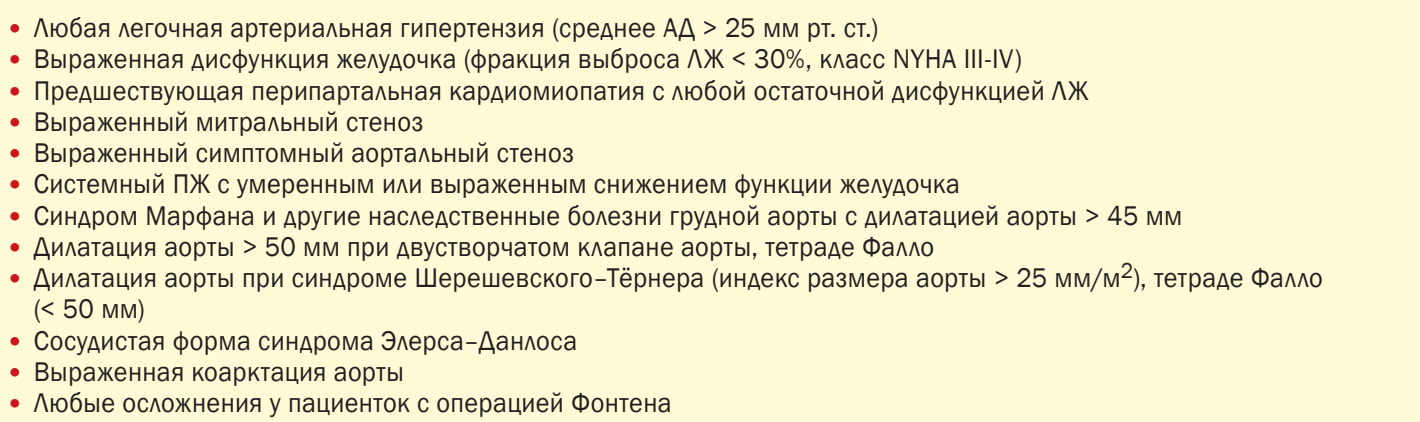 \\
\hline
\end{tabular}


такие как фракция выброса левого желудочка (ФВЛЖ), площадь клапанного отверстия.

Риск оценивают не только перед первой, но и каждой последующей беременностью. Необходимость переоценки понятна, так как состояние сердца может измениться и во время наступившей беременности, и после нее в силу разных обстоятельств.

\section{Структурно-функциональные изменения сердца при нормально протекающей беременности}

Первое ЭхоКГ-обследование беременной обычно проводят при сроке 12 недель и в последующих триместрах повторяют по мере необходимости при наличии показаний [2].

Во время беременности у здоровой женщины объем жидкости увеличивается на $40-100 \%$, а объем циркулирующей крови - на 30-50\%, что неизбежно приводит к увеличению преднагрузки [4]. Первые изменения можно определить при ЭхоКГ уже на 5-й неделе гестации, когда определяется увеличение ударного объема, сердечного выброса в среднем на 20\%. К 20-й неделе гестации сдвиги увеличиваются, и прирост этих показателей может быть на 30-50\% выше по сравнению с начальными. К концу І триместра отчетливо регистрируется увеличение размеров левого предсердия, к концу II триместра - возможно увеличение размеров и объемов левого желудочка, которые постепенно увеличиваются вплоть до родов [5]. В ІІІ триместре увеличивается индекс сферичности ЛЖ. При сравнении показателей в наибольшей степени увеличиваются объемы ЛЖ - на 30-50\%, в меньшей степени его размеры - в среднем на $11 \%$ [5]. При этом они не выходят за пределы общепринятой нормы.

В эти же сроки описывается «физиологическая гипертрофия беременных» (pregnancy-induced hypertrophy), при которой увеличивается толщина стенок ЛЖ и масса миокарда. Максимальная масса миокарда определяется в III триместре беременности. Общий прирост массы миокарда иногда составляет до 70-80\% по отношению к исходной [6, 7]. Важно, что нарушений функции сердца при этом не возникает.

В I триместре допплерография может выявлять увеличение скорости раннего диастолического наполнения ( $\mathrm{Ve})$ и снижение скорости кровотока в систолу предсердий

\begin{tabular}{|c|}
\hline $\begin{array}{l}\text { Предикторы риска осложнений у матери с Таблица } 2 \\
\text { кардиоваскулярной патологией* / Predictors of the risk } \\
\text { of complications in a mother with cardiovascular disease* }\end{array}$ \\
\hline $\begin{array}{l}\text { Сердечно-сосудистые события в анамнезе (сердечная недостаточ- } \\
\text { ность, транзиторная ишемическая атака, инсульт Ао беременности } \\
\text { или аритмии) }\end{array}$ \\
\hline Исходный функциональный класс XCH III-IV по NYHA \\
\hline 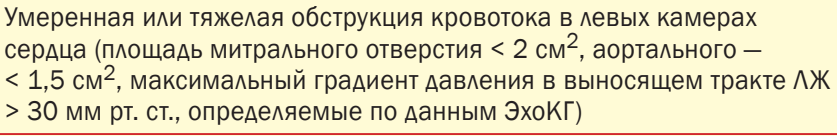 \\
\hline Снижение систолической функции АЖ (фракция выброса < 40\%) \\
\hline ^егочная гипертензия \\
\hline Уровень натрийуретического пептида > 128 пг/м^ \\
\hline Курение \\
\hline Механические протезы клапанов сердца \\
\hline Оперированный или неоперированный цианотический порок сердца \\
\hline $\begin{array}{l}\text { Примечание. * На основании исслеАований CARPREG, ZAHARA, } \\
\text { ROPAC. }\end{array}$ \\
\hline
\end{tabular}

(Va) трансмитрального диастолического потока. В конце II и III триместре возрастает вклад сокращения левого предсердия в наполнение левого желудочка, что отражается в увеличении скорости Va и, соответственно, относительном снижении отношения Е/А. Удлиняется время изоволюметрического сокращения (IVRT) и время замедления потока (DT), но время ускорения (AT) остается стабильным [7]. B I триместре немного нарастает скорость систолического и одновременно снижается скорость диастолического кровотока в легочных венах. Важно, что эти изменения происходят у беременных с нормальным АД или незначительно повышенным диастолическим АД вследствие экспрессии фетальных генов, физиологических гормональных сдвигов. Описано снижение систолической продольной деформации базальных, срединных и апикальных сегментов левого желудочка и систолической деформации правого желудочка, которые выявляются при более углубленном исследовании с использованием режимов strain, strain-rate. Вместе с этим никаких существенных изменений радиальной и циркулярной деформации желудочков не регистрируют [8]. Следует отметить, что снижение это незначительно, кратковременно, не сопровождается какими-либо клиническими симптомами и самопроизвольно проходит без лечения.

Систолическое давление в легочной артерии увеличивается иногда до 40 мм рт. ст. Немного, на 2-3 мм, увеличивается размер корня аорты. Возможно появление митральной и трикуспидальной регургитации 1-2 степени [1].

Все эти изменения обратимы. Полная нормализация отмечается к 3-6 месяцам после родоразрешения. Описаны наблюдения, в которых восстановление регистрировалось только через 1 год.

\section{Сердечная гемодинамика при многоплодной беременности}

Сердечная гемодинамика при одноплодной и многоплодной беременности имеет ряд отличий. Это связано с более высокими показателями уровней периферического сосудистого сопротивления артериальному кровотоку в плодово-плацентарной циркуляции, что можно рассматривать как адаптационную реакцию в условиях ее повышенного функционального напряжения при многоплодии. Если в I триместре беременности показатели маточно-плацентарной гемодинамики при многоплодной и одноплодной беременностях существенно не различаются, то в дальнейшем происходит интенсивное снижение сосудистой резистентности кровотока в маточных артериях, что приводит к изменению гемодинамических показателей, которые в большей степени выражены при многоплодной беременности. Так, масса ЛЖ при беременности увеличивается примерно на $50 \%$ из-за увеличения конечно-диастолического и конечно-систолического его размера.

При многоплодной беременности объем циркулирующей крови стремительно нарастает во II-III триместрах беременности и к 34-й неделе увеличивается на 50-70\% от исходных значений. Подобные изменения ведут к значительному увеличению работы сердца, при этом ударный объем сердца увеличивается более чем на $30 \%$, частота сердечных сокращений возрастает на $15-20 \%$, а общее периферическое сопротивление уменьшается примерно на $25 \%$.

\section{Беременные с артериальной гипертензией}

Выделяют следующие клинические варианты АГ при беременности: 
- АГ, имевшаяся до беременности;

- хроническая АГ;

- гипертоническая болезнь или симптоматическая АГ;

- гестационная АГ;

- хроническая АГ, осложнившаяся преэклампсией;

- преэклампсия/эклампсия.

Определение или уточнение поражения сердца как органа-мишени при АГ, то есть выявление гипертрофии ЛЖ в совокупности с оценкой систолической и диастолической функции миокарда, являются первоочередными задачами ЭхоКГ при ведении больных с АГ и необходимы для получения дополнительной информации о прогнозе и риске. При выявлении гипертрофии необходимо проводить дифференциальный диагноз с физиологической гипертрофией миокарда (pregnancy-induced hypertrophy), которая выявляется со II триместра, и эта гипертрофия носит, как правило, концентрический характер. Кроме того, данные ряда исследований демонстрируют, что во время беременности, осложненной гипертонией, масса миокарда левого желудочка и относительная толщина стенок увеличиваются больше, чем во время нормотензивной беременности [9].

\section{Перипартальная кардиомиопатия}

Заболевание, которое возникает исключительно во время беременности или в послеродовом периоде (в интервале между последними месяцами беременности и сроком до 5 месяцев после родов). Данные о ее частоте противоречивы: от 1 случая на 3000-4000 беременностей до 1 случая на 15000 родов [10], что, возможно, свидетельствует о трудностях диагностики. Данную патологию подозревают во всех случаях, когда у беременной появляются признаки сердечной недостаточности, нарушения ритма сердца, а также если состояние после родов медленно нормализуется. Риск возникновения перипартальной кардиомиопатии увеличен у женщин старше 30 лет при многоплодной беременности, преэклампсии, у больных с бронхиальной астмой, анемией.

ЭхоКГ имеет приоритетное значение в выявлении перипартальной кардиомиопатии. Диагноз устанавливают на основании совокупности клинических и ЭхоКГ-критериев [10, 11]:

1. Сердечная недостаточность:

- последний месяц беременности;

- 5 месяцев после родов.

2. Отсутствие предшествующих болезней сердца.

3. Отсутствие определенных причин болезни, кроме беременности.

4. Явные ЭхоКГ-признаки дисфункции ЛЖ:

- фракция выброса менее 45\% и/или фракционное укорочение менее $30 \%$;

- конечный диастолический диаметр ЛЖ больше 2,7 см/м².

При сниженной фракции выброса $(<35 \%)$ в полости ЛЖ образуются тромбы, являющиеся причиной тромбоэмболий.

Выздоровление и возврат к норме достигается у большинства, но не у всех женщин, и в ряде случаев функция сердца имеет положительную динамику, но только до определенных значений фракции выброса. При неполном выздоровлении («выздоровление с дефектом») перипартальная кардиомиопатия может вновь возникать при последующих беременностях [10].

Таким образом, диагноз перипартальной кардиомиопатии ставится методом исключения при отсутствии признаков всех других возможных причин систолической недостаточности. Вот почему в каждом случае необходима очень внимательная ЭхоКГ-оценка структуры и функции сердца, сопоставление полученных данных с предыдущими (наилучшее - с протоколом ЭхоКГ до беременности), чтобы дифференцировать имеющуюся картину с изменениями, происходящими при тяжелой преэклампсии, других формах кардиомиопатий, при врожденной и приобретенной патологии сердца, а также с тромбоэмболией легочной артерии, инфарктом миокарда, внутрисердечными образованиями, сепсисом.

\section{Беременные с пороками сердца}

В структуре всех СС3 у беременных, по разным данным, $30-50 \%$ составляют ВПС, около $15 \%$ - ППС [12, 13].

Установление диагноза, оценка анатомии клапана, гемодинамики, степени тяжести порока, в том числе с точки зрения показаний к кардиохирургической коррекции во время беременности (например, баллонной комиссуротомии), наблюдение беременных в послеоперационном периоде вот задачи, которые стоят перед ЭхоКГ-исследованием [2].

В последние десятилетия значительно возросло число беременных с ВПС, благодаря широкому внедрению кардиохирургических методов коррекции. Прооперированные в раннем детстве девочки достигают детородного возраста. Их клиническое состояние, качество жизни часто бывают вполне удовлетворительными для того, чтобы планировать беременность.

Первоначально беременные с ранее подтвержденными ВПС должны быть обследованы эхокардиографически при сроке до 10-12 недель беременности для определения прогноза возможности вынашивания беременности [2]. По результатам обследования коллегиально вместе с кардиологом, акушером-гинекологом и при необходимости - кардиохирургом выносится заключение о наличии или отсутствии противопоказаний к беременности, необходимости кардиохирургического лечения.

Далее при нормальном течении беременности, отсутствии риска или низком риске ЭхоКГ выполняют в 18-22 и 27-32 недели, а также перед родами. Большая кратность ЭхоКГ определяется клиническим состоянием, типом порока сердца. Зачастую именно ЭхоКГ-заключение является решающим в вопросах дальнейшей тактики ведения беременности и досрочного родоразрешения по медицинским показаниям. Кесарево сечение целесообразно пациенткам с острой рефрактерной сердечной недостаточностью, тяжелым аортальным стенозом, тяжелой формой легочной гипертонии (включая синдром Эйзенменгера) или острой сердечной недостаточностью.

\section{Митральный стеноз}

В структуре ППС у беременных митральный стеноз занимает первое месте (около 60\%) и является наиболее опасным, с ним связаны серьезные, иногда фатальные осложнения [14]. Нередко данный порок впервые диагностируют именно во время беременности. Это связано с тем, что он длительное время остается бессимптомным. При площади митрального отверстия больше $1,5 \mathrm{~cm}^{2}$ течение беременности близко к обычному. Однако умеренный и тяжелый стеноз (площадь клапанного отверстия меньше $1,5 \mathrm{~cm}^{2}$ ) ассоциирован с клиническими проявлениями (прежде всего одышкой) и высоким риском таких осложнений, как отек легких, нарушения ритма сердца, прогрессирующая сердечная недостаточность.

ЭхоКГ- оценка морфологии клапана у беременных с митральным стенозом имеет особое значение. От того, каковы изменения клапана, есть ли кальциноз створок, зависит необходимость проведения баллонной митральной 
комиссуротомии. В настоящее время эта операция выполняется успешно при любом сроке беременности, при этом общая материнская летальность не превышает $1 \%$ (несколько выше, до 2\%, при кальцинозе створок), гибель плода составляет около $2 \%$, а уровень послеоперационных осложнений низкий. В то же время при открытой комиссуротомии и протезировании клапана гибель плода возникает в $10-30 \%$ случаях. Кроме того, при установке клапанных протезов в дальнейшем будет требоваться прием антикоагулянтов [15].

Кратность проведения ЭхоКГ зависит от тяжести митрального стеноза, состояния гемодинамики. При легком митральном стенозе, стабильном состоянии беременной обследования выполняют в каждом триместре и перед родами. При умеренном и тяжелом - не реже одного раза в два месяца или ежемесячно [2, 14].

\section{Митральная недостаточность}

Недостаточность митрального клапана у беременных обычно бывает ревматического генеза, а также обусловленная пролапсом митрального клапана, в том числе вследствие миксоматозной дегенерации. Встречается также функциональная регургитация при врожденных пороках сердца, в том числе оперированных в детском возрасте.

В отличие от митрального стеноза, осложнения у беременных с этим пороком нечасты, что объясняется хорошей адаптацией к нагрузке объемом в результате снижения общего периферического сосудистого сопротивления при беременности и, соответственно, уменьшения объема регургитации.

При ЭхоКГ устанавливают степень тяжести регургитации, размеры полостей сердца. При планировании беременности пациенткам со среднетяжелой и тяжелой регургитацией рекомендуется выполнение пробы с физической нагрузкой.

Во время беременности почти во всех случаях отмечается нарастание степени регургитации, размеров левых полостей сердца. Риск осложнений зависит от тяжести регургитации и функции ЛЖ. Заметим, что митральная регургитация препятствует образованию тромбов в левых отделах сердца. При легкой и среднетяжелой регургитации ЭхоКГ повторяют каждый месяц, при тяжелой - не реже одного раза в 2 месяца [2, 14].

\section{Стеноз аортального клапана}

При планировании беременности рекомендовано определить степень аортального стеноза, а также уточнить адаптационный резерв сердечно-сосудистой системы, проведя пробу с физической нагрузкой. Так, для легкого аортального стеноза характерна хорошая адаптация к нагрузке объемом, отсутствие симптомов, благоприятное течение беременности. Напротив, при тяжелом аортальном стенозе адаптация сердца к нагрузке намного хуже, но, несмотря на это, также возможно благоприятное течение беременности. При ЭхоКГ у планирующих беременность пациенток с аортальным стенозом фиксируют внимание на функции ЛЖ. При нарушенной систолической функции, согласно рекомендациям, целесообразна вальвулопластика или протезирование аортального клапана всем пациенткам с тяжелым аортальным стенозом с клиническими симптомами, а также с бессимптомным пороком, но нарушенной функцией ЛЖ или снижением толерантности к физической нагрузке. В противном случае беременность не рекомендуется.

При ЭхоКГ в случаях аортального стеноза отмечается увеличение размеров ЛЖ и предсердия, среднего и максимального градиента давления от триместра к триместру [16]. Площадь аортального отверстия, фракция выброса, как правило, не изменяются.

ЭхоКГ выполняют при тяжелом аортальном стенозе ежемесячно, в остальных случаях - один раз в триместр [2, 14].

\section{Аортальная недостаточность}

При тяжелой аортальной регургитации с дисфункцией ЛЖ и острой аортальной регургитации беременность переносится плохо. Поэтому таким пациенткам необходимо обследование до беременности. Акцентируют внимание на определении степени тяжести регургитации, измерении размеров и функции ЛЖ, размеров аорты. При выявлении тяжелой регургитации в сочетании с нарушением функции или дилатацией ЛЖ нужна коррекция порока до наступления беременности [16] в связи с высоким риском развития сердечной недостаточности. ЭхоКГ пациенткам с легкой и среднетяжелой регургитацией проводят каждые 3 месяца, но при тяжелой регургитации периодичность устанавливают индивидуально, исходя из состояния женщины и течения беременности $[2,14]$.

\section{Трикуспидальная недостаточность}

Трикуспидальная регургитация у беременных в абсолютном большинстве случаев бывает функциональной вследствие перегрузки правого желудочка давлением и/или объемом. Однако при выраженной дилатации клапанного кольца ( $\geqslant 40$ мм), среднетяжелой и тяжелой трикуспидальной регургитации пациентку следует консультировать с кардиохирургами для решения вопроса об оперативном вмешательстве. При планировании беременности у пациенток с тяжелой регургитацией рекомендована операция до беременности.

\section{Аилатация восходящей аорты}

Дилатация восходящей аорты выявляется примерно у $50 \%$ беременных с бикуспидальным аортальным клапаном. В этих случаях особо внимательно контролируют размеры аорты при каждом ЭхоКГ-обследовании [17]. Если у женщины, планирующей беременность, диаметр восходящей аорты $\geqslant 50$ мм (27,5 мм/м²), то независимо от наличия симптомов нужно обсуждать возможность оперативного вмешательства до беременности, так как степень дилатации может увеличиваться и есть риск ее диссекции. Риск расслоения стенки аорты выше при синдроме Марфана, Элерса-Данлоса.

Женщинам с расширением корня аорты свыше 40 мм, дилатацией восходящей аорты рекомендовано проводить ЭхоКГ каждые 4-8 недель и через 6 месяцев после родов [17]. Нарастание размера во время беременности считают фактором риска диссекции аорты. Пациенткам с диаметром аорты более 45 мм, с синдромом Марфана, у которых диаметр аорты составляет 40-45 мм, острой или хронической расслаивающей аневризмой аорты целесообразно оперативное родоразрешение.

\section{Заключение}

Таким образом, ЭхоКГ-исследование является необходимым для оценки структурно-функционального состояния сердца во время беременности, особенно у женщин с сердечно-сосудистой патологией в анамнезе. Кратность обследования и алгоритм индивидуальны и зависят от тяжести сопутствующей патологии [18]. Правильная интерпретация полученных ЭхоКГ-изменений позволяет врачу сделать вывод о нормальном либо патологическом воздействии 
на сердце происходящих изменений в организме женщины. Проведение ЭхоКГ является обязательным для оценки материнского риска, определения показаний/противопоказаний к вынашиванию беременности, а также способа родоразрешения.

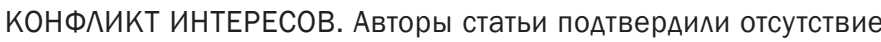
конфликта интересов, о котором необходимо сообщить.

CONFLICT OF INTERESTS. Not declared.

\section{Митература/References}

1. 2018 ESC Guidelines for the management of cardiovascular diseases during pregnancy // European Heart Journal. 2018; 39 (34): 3165-3241. DOI: 10.1093/eurheartj/ehy340ESC.

2. Диагностика и лечение сердечно-сосудистых заболеваний при беременности 2018. Национальные рекомендации. Разработаны Комитетом экспертов Российского кардиологического общества (РКО). Секция заболевания сердечно-сосудистой системы у беременных // Российский кардиологический журнал. 2018; 3 (155) 91-134.

[Diagnostika i lechenie serdechno-sosudistykh zabolevanii pri beremennosti 2018. Natsional'nye rekomendatsii.

Razrabotany Komitetom ekspertov Rossiiskogo

kardiologicheskogo obshchestva (RKO). Sektsiia zabolevaniia serdechno-sosudistoi sistemy u beremennykh [Diagnosis and treatment of cardiovascular diseases during pregnancy 2018. National guidelines. Developed by the Committee of experts of the Russian society of cardiology (RSC). Section of cardiovascular diseases in pregnant women] // Rossiiskii kardiologicheskii zhurnal. 2018; 3 (155): 91-134. (In Russian.)] DOI: 10.15829/1560-4071-2018-3-91-134.

3. Tsiaras S., Poppas A. Cardiac disease in pregnancy: value of echocardiography // Curr Cardiol Rep. 2010; 12 (3): 250-256. DOI: 10.1007/s11886-010-0106-9.

4. Narayanan M., Elkayam U., Naqvi T. Z. Echocardiography in Pregnancy: Part 2 // Curr Cardiol Rep. 2016; 18 (9): 90. DOI: $10.1111 /$ echo.13242.

5. Savu O., Jurcuţ R., Giuşcă S., van Mieghem T. et al. Morphological and functional adaptation of the maternal heart during pregnancy // Circ Cardiovasc Imaging. 2012; 5 (3): 289-297. DOI: 10.1161/CIRCIMAGING.111.970012.

6. Li J., Umar S., Amjedi M., Iorga A., Sharma S., Nadadur R. D., Regitz-Zagrosek $V$., Eghbali M. New frontiers in heart hypertrophy during pregnancy // Am J Cardiovasc Dis. 2012; 2 (3): 192-207.

7. Schannwell C. M., Zimmermann T., Schneppenheim M., Plehn G., Marx R., Strauer B. E. Left Ventricular

Hypertrophy and Diastolic Dysfunction in Healthy Pregnant Women // Cardiology. 2002; 97: 73-78.

DOI: $10.1159 / 000057675$.

8. Cong J., Fan T., Yang X., Squires J. W., et al. Structural and functional changes in maternal left ventricle during pregnancy: a three-dimensional speckle-tracking echocardiography study // Cardiovasc Ultrasound. 2015; $13: 6$. DOI:10.1186/1476-7120-13-6.

9. De Haas S., Ghossein-Doha C., Geerts L., van Kuijk S. M. J., van Drongelen J., Spaanderman M. E. A. Cardiac remodeling in normotensive pregnancy and in pregnancy complicated by hypertension: systematic review and meta-analysis // Ultrasound Obstet Gynecol. 2017; 50 (6): 683-696. DOI: $10.1002 /$ uog. 17410.

10. Arany Z. Understanding Peripartum Cardiomyopathy // Annu Rev Med. 2018; 69: 165-176. DOI: 10.1146/annurevmed-041316-090545.

11. Bauersachs J., Arrigo M., Hilfiker-Kleiner D., Veltmann C., et al. Current management of patients with severe acute peripartum cardiomyopathy: practical guidance from the Heart Failure Association of the European Society of Cardiology Study Group on peripartum cardiomyopathy // Eur J Heart Fail. 2016; 18 (9): 1096-105. DOI: 10.1002/ejhf.586.

12. Nanna M., Stergiopoulos K. Pregnancy Complicated by Valvular Heart Disease: An Update // Journal of the American Heart Association. June 2014, Wolters Kluwer Health. DOI: 10.1161/jaha.113.000712.

13. Pessel C., Bonanno C. Valve disease in pregnancy // Semin Perinatol. 2014; 38 (5): 273-284. S0146-0005(14)000342.10.1053/j.semperi.2014.04.016.

14. Leśniak-Sobelga A., Tracz W., Kostkiewicz M., Podolec P., Pasowicz M. Clinical and echocardiographic assessment of pregnant women with valvular heart diseases- maternal and fetal outcome // Int J Cardiol. 2004; 94 (1): 15-23. OpenUrl CrossRef PubMed.

15. Roeder H. A., Kuller J. A., Barker P. C., James A. H. Maternal valvular heart disease in pregnancy // Obstet Gynecol Surv. 2011; 66 (9): 561-571. DOI:10.1097/OGX.0b013e318238605d.

16. Windram J. D., Colman J. M., Wald R. M., Udell J. A., Siu S. C., Silversides $C$. K. Valvular heart disease in pregnancy // Best Pract Res Clin Obstet Gynaecol. 2014; 28 (4): 507-518. DOI: 10.1016/j.bpobgyn.2014.03.009.

17. Shim W. J. Role of echocardiography in the management of cardiac disease in women // J Cardiovasc Ultrasound. 2014; 22 (4): 173-179. DOI: 10.4250/jcu.2014.22.4.173.

18. Горохова С. Г., Морозова Т. Е., Аракеляни А. А., Барабанова Е. А., Дьяконова Е. Г. Алгоритм эхокардиографического исследования у беременных // Российский кардиологический журнал. 2018; 23 (12): 75-82.

[Gorokhova S. G., Morozova T. E., Arakeliants A. A., Barabanova E. A., D'iakonova E. G. Algoritm ekhokardiograficheskogo issledovaniia u beremennykh/ Algorithm of echocardiography in pregnant women // Russian Journal of Cardiology. 2018; (12): 75-83.

(In Russian.)] 